\title{
Geranylgeranyl Diphosphate Synthase Modulates Fetal Lung Branching Morphogenesis Possibly through Controlling K-Ras Prenylation
}

\author{
Wen-Jun Jia, ${ }^{* \dagger}$ Shan Jiang, ${ }^{*}$ Qiao-Li Tang, ${ }^{*}$ Di Shen,${ }^{*}$ Bin Xue, ${ }^{*}$ Wen Ning, ${ }^{\ddagger}$ and Chao-Jun Li ${ }^{*}$
}

From the Ministry of Education Key Laboratory of Model Animal for Disease Study, * the School of Medicine and Model Animal Research Center of Nanjing University, Nanjing; the Department of Hepatopancreatobiliary Surgery, ${ }^{\dagger}$ the Affiliated Drum Tower Hospital of Medical School of Nanjing University, Nanjing; and State Key Laboratory of Medicinal Chemical Biology, ${ }^{\ddagger}$ the College of Life Sciences, Nankai University, Tianjin, China

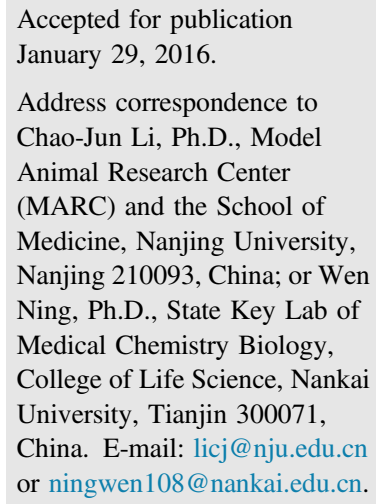

\begin{abstract}
$\mathrm{G}$ proteins play essential roles in regulating fetal lung development, and any defects in their expression or function (eg, activation or posttranslational modification) can lead to lung developmental malformation. Geranylgeranyl diphosphate synthase (GGPPS) can modulate protein prenylation that is required for protein membrane-anchoring and activation. Here, we report that GGPPS regulates fetal lung branching morphogenesis possibly through controlling K-Ras prenylation during fetal lung development. GGPPS was continuously expressed in lung epithelium throughout whole fetal lung development. Specific deletion of geranylgeranyl diphosphate synthase 1 (Ggps1) in lung epithelium during fetal lung development resulted in neonatal respiratory distress syndrome-like disease. The knockout mice died at postnatal day 1 of respiratory failure, and the lungs showed compensatory pneumonectasis, pulmonary atelectasis, and hyaline membranes. Subsequently, we proved that lung malformations in Ggps1-deficient mice resulted from the failure of fetal lung branching morphogenesis. Further investigation revealed Ggps1 deletion blocked K-Ras geranylgeranylation and extracellular signal-related kinase 1 or 2 /mitogen-activated protein kinase signaling, which in turn disturbed fibroblast growth factor 10 regulation on fetal lung branching morphogenesis. Collectively, our data suggest that GGPPS is essential for maintaining fetal lung branching morphogenesis, which is possibly through regulating K-Ras prenylation. (Am J Pathol 2016, 186: 1454-1465; http://dx.doi.org/ 10.1016/j.ajpath.2016.01.021)
\end{abstract}

Neonatal respiratory distress syndrome (NRDS) is a critical clinical problem caused by pulmonary surfactant insufficiency and structural immaturity in the lungs of premature infants. ${ }^{1}$ However, the correlation between lung development and neonatal lung disease such as NRDS is still incompletely understood. In mice, lung development initiates at embryonic day (E)9.5, then is divided into embryonic period (E9.5 to E14.2), pseudoglandular period (E14.2 to E16.5), canalicular period (E16.5 to E17.5), saccular period [E17.5 to postnatal day (P5)], and alveolar period (P5 to P30). ${ }^{2}$ Through these periods, the set of out-pouches in the foregut converts to a fluid-filled lung, accompanied by complex airway branching and lung epithelial differentiation with angiogenesis. ${ }^{3}$ This process includes outgrowth, elongation, differentiation, and subdivision, which is named branching morphogenesis. ${ }^{4}$ However, branching morphogenesis does not continue postnatally, the lung merely increases the airways' length and diameter to enlarge the size. ${ }^{5}$ Thus, the formation, distribution, and differentiation of the epithelium in branching morphogenesis during fetal lung development are critical to postnatal respiratory maturation and function, and as the future bronchial tree is formed in the pseudoglandular period, branching morphogenesis in this period is particular important.

Supported by the National Basic Research Program of China 973 program grant 2012CB524900 (C.-J.L.), National Science and Technology Supplementary Program of China grant 2011BAI15B02 (C.-J.L.), National Natural Science Foundation of China grants 31171306 and 31371373 (B.X.), and Nature Science Foundation of Jiangsu Province grant BK2011568 (B.X.).

W.-J.J. and S.J. contributed equally to this work.

Disclosures: None declared. 
Fibroblast growth factor 10 (FGF10) is implicated in mouse lung branching morphogenesis, which is mainly expressed in distal mesenchyme, and exerts a modulating effect on distal epithelial cell elongation. ${ }^{6,7}$ FGF10 can bind to the fibroblast growth factor receptor 2 (FGFR2), and thus activates K-Ras/ mitogen-activated protein kinase (MAPK) signaling, which can induce the expression of Sprouty 2 in epithelium. Then Sprouty2, in turn, suppresses distal epithelial cell outgrowth by blocking the FGF10 signals. ${ }^{8}$ This dynamic interplay between FGF10 and Sprouty2 modulates the fetal lung branching morphogenesis. ' Meanwhile, an early study that used $S P C$-rtTA/TetO-Cre/Kras ${ }^{L S L-G 12 D}$ mutant mice reported that overactivation of K-Ras in fetal lung epithelium led to a profound defect in lung branching morphogenesis by constitutively activating extracellular signal-related kinase 1 or 2(Erk1/2)/MAPK signaling, thus resulting in a striking upregulation of Sprouty2. ${ }^{10}$ Moreover, with the use of the highly specific inhibitor of MAPK kinase $1 / 2$, the fetal lung malformation in Shh-Cre/Kras ${ }^{L S L-G 12 D}$ mice could be reversed at approximately E11.5. ${ }^{11}$ Thus, the properly activated K-Ras/MAPK signaling and the mediated interplay between FGF10 and Sprouty 2 are irreplaceable in branching morphogenesis during fetal lung development.

Functional K-Ras needs to undertake prenylation to ensure its membrane association and activation, which is regulated by the availability of its substrates, geranylgeranyl diphosphate (GGPP) and farnesyl diphosphate (FPP). ${ }^{12}$ GGPP synthase (GGPPS) tilts intracellular FPP and GGPP by synthesizing GGPP from FPP. We have previously reported that GGPPS-mediated protein prenylation regulates multiple physiologic processes, including male fertility, ${ }^{13}$ heart growth, ${ }^{14}$ liver injury and cancer, ${ }^{15,16}$ and metabolic homeostasis. ${ }^{17,18}$ Interestingly, GGPPS could also mediate the cigarette smoke-induced lung inflammation by controlling K-Ras prenylation. ${ }^{19,20}$ However, whether GGPPScontrolled protein prenylation is involved in the regulation of fetal lung development is unclear.

Here, we evaluated the function of GGPPS on branching morphogenesis in fetal lung development by specific deletion of Ggps1 in fetal lung epithelium. Our data showed that Ggps1 deficiency in lung epithelium hampered the fetal lung branching morphogenesis and led to prenatal lung malformation and NRDS-like disease. Furthermore, we proposed a potential mechanism that Ggpsl deletion abrogated the interplay between FGF10 and Sprouty2 by interfering with K-Ras geranylgeranylation and Erk1/2/MAPK signaling pathway. Collectively, GGPPS plays an essential role in regulating fetal lung branching morphogenesis, which is possibly through mediating FGF10 signaling by controlling K-Ras prenylation.

\section{Materials and Methods}

\section{Antibodies and Reagent}

Primary antibodies against GGPPS (for Western blot analysis), Sprouty2, surfactant protein (SP)-B, SP-C, K-Ras, and horseradish peroxidase-tagged secondary antibody were purchased from Santa Cruz Biotechnology (Santa Cruz, CA). Antibodies against total Erk1/2, total Akt, and their phosphorylated forms were from Cell Signaling Technology (Danvers, MA). GGPPS antibody used for immunochemistry was from ProteinTech (Chicago, IL), and Ki-67 was from Abcam (Cambridge, MA). Doxycycline (Dox) and DAPI were from Sigma-Aldrich (St. Louis, MO).

\section{Animals}

C57BL/6J mice and SPC-rtTA and TetO-Cre mice were purchased from Model Animal Research Center of Nanjing University. Ggps $1^{\text {flox/flox }}$ mice were generated through homologous recombination as previously described. ${ }^{13}$ The inducible lung epithelium-specific GGPS1 knockout mice $\left(S P C-r t T A / T e t O-C r e / G g p s l^{\text {flox/flox }}\right)$ were generated by crossing Ggps $1^{\text {flox/flox }}$ mice with SPC-rtTA mice and TetOCre mice. All mice were backcrossed for at least six generations to $C 57 B L / 6 J$ strain background and were genotyped by genomic DNA PCR. Mice were housed in a specific pathogen-free condition with 12-h light/12-h dark cycle in a temperature- and humidity-controlled environment and fed ad libitum. Administration of $1 \mathrm{mg} / \mathrm{mL}$ Dox was started from E7.5 or E16.5 by feeding the pregnant mice with drinking water. All animal procedures were performed in accordance with the Animal Care and Use Committee at the Model Animal Research Center of Nanjing University, Nanjing, China.

\section{Histologic Analysis, H\&E Staining, and Periodic Acid-Schiff Staining}

Embryonic or neonatal lung was isolated and washed twice with phosphate-buffered saline (PBS). Then the gross view of lung was visualized by stereomicroscope, and the number of branches was counted manually. For histologic analysis, lungs of the indicated ages were isolated and fixed in $4 \%$ paraformaldehyde at $4{ }^{\circ} \mathrm{C}$ for 24 hours and then processed to paraffin embedding and sectioned at $5 \mu \mathrm{m}$. The hematoxylin and eosin (H\&E) staining and periodic acid-Schiff staining were performed according to the standard protocols. ${ }^{21}$

\section{PC and PG Contents}

Lung tissue phosphotidyl choline (PC) and phosphatidylglycerol (PG) contents of wild-type (WT) and lung-specific Ggps1 knockout mice at P1 were measured according to Fitzgerald et al. ${ }^{22}$ Briefly, after homogenization, total lung lipids were isolated from lungs by one chloroform-methanol $(1: 1, \mathrm{v} / \mathrm{v})$ extraction and two chloroform extractions. Then, the organic phases were washed once with $1 \mathrm{~mol} / \mathrm{L} \mathrm{KCl}$ and twice with water, and dried under nitrogen gas. Then samples were introduced with an autosampler (SIL-20AHT; Shimadzu, Kyoto, Japan) and subjected to automated electrospray ionization-tandem mass spectrometry (LCMS-8040; Shimadzu) analysis. The internal standards used were 
$0.66 \mathrm{nmol}$ of di14:0-PC, $0.66 \mathrm{nmol}$ of di24:1-PC, $0.66 \mathrm{nmol}$ of 13:0-lyso PC, $0.66 \mathrm{nmol}$ of 19:0-lyso PC, $0.36 \mathrm{nmol}$ of di14:0-PG, $0.36 \mathrm{nmol}$ of di24:1-PG, $0.36 \mathrm{nmol}$ of 14:0-lyso $\mathrm{PG}$, and $0.36 \mathrm{nmol}$ of 18:0-lyso PG.

Immunohistochemistry, Immunofluorescence, and TUNEL Assay

The expression pattern of GGPPS in embryonic lungs was detected in $C 57 B L / 6 \mathrm{~J}$ mice by immunohistochemistry, and the localization of epithelial-specific marker SP-C and Ki-67 staining of proliferative cells was measured by immunofluorescence, as previously described, ${ }^{13,14}$ in WT and fetal lung epithelium-specific Ggps1 knockout mice lungs. In situ DNA fragmentation was assessed by using the DeadEnd Colorimetric TUNEL (terminal deoxynucleotidyl transferasemediated dUTP nick-end labeling) System (Promega, Madison, WI), according to the manufacturer's instructions.

\section{Subcellular Fractionation}

To assess the K-Ras membrane-anchoring, subcellular fractionation of the embryonic lungs was performed with the Triton X-114 partition method. ${ }^{23}$ Briefly, six E13.5 embryonic lungs were lyzed. Then total protein concentration was diluted to $1 \mathrm{mg} / \mathrm{mL}$ and partitioned with the same volume of $4 \%$ Triton X-114 for 5 minutes at $37^{\circ} \mathrm{C}$ to solubilize and fractionate the lipid-rich cell membrane. Then the subcellular fractions were immunoprecipitated with the K-Ras antibody and were subjected to Western blot analysis.

\section{Immunoprecipitation and Western Blot Analysis}

Immunoprecipitation was performed according to a standard protocol. $^{20} \mathrm{~K}$-Ras antibody was used to form immune complex with K-Ras proteins in lysates and was immunoprecipitated down with protein A/G plus agarose. Finally, the equivalent protein samples were subjected to Western blot analysis against K-Ras.

\section{RNA Extraction and Real-Time PCR}

RNA was prepared with the TRIzol reagent (Takara Bio, Tokyo, Japan) and reversed transcribed with PrimeScript RT reagent (Takara Bio) according to the manufacturer's protocol. Real-time PCR was performed on an ABI-7300 (Applied Biosystems, Carlsbad, CA) to detect the gene expression. All quantitation was performed in triplicate and was normalized to internal 18-strand RNA. The sequence of primers are as follows: GGPPS, forward, 5'-TTTTGCATACACTCGACACACT- $3^{\prime}$, and reverse, 5'-GGCCTCAATTTGTTTGTAGGCT- $3^{\prime}$; ion/water channel protein (AQP5), forward, 5'-AGAAGGAGGTGTGTTCAGTTGC-3', and reverse, 5'-GCCAGAGTAATGGCCGGAT-3'; bone morphogenetic protein 4 (BMP4), forward, $5^{\prime}$-TTCCTGGTAACCGAATGCTGA-3', and reverse, 5'-CCTGAAT-
CTCGGCGACTTTTT-3'; Clara cell-specific protein (CCSP), forward, 5'-ATGAAGATCGCCATCACAATCAC-3', and reverse, 5'-GGATGCCACATAACCAGACTCT-3'; FGF10, forward, 5'-TTTGGTGTCTTCGTTCCCTGT- $3^{\prime}$, and reverse, 5'-TAGCTCCGCACATGCCTTC-3'; forkhead box protein J1 (FoxJ1), forward, $5^{\prime}$-CCCTGACGACGTGGACTATG-3', and reverse, 5'-GCCGACAGAGTGATCTTGGT-3'; SP-B, forward, 5'-CTGTGCCAAGAGTGTGAGGA-3', and reverse, 5'-TTGGGGTTAATCTGGCTCTG-3'; sonic hedgehog protein (Shh), forward, 5'-AAAGCTGACCCCTTTAGCCTA-3', and reverse, 5'-TTCGGAGTTTCTTGTGATCTTCC-3'; Sprouy2, forward, 5'-TCCAAGAGATGCCCTTACCCA-3', and reverse, 5'-GCAGACCGTGGAGTCTTTCA-3'; and $18 \mathrm{~S}$, forward, $5^{\prime}$-GTCTGTGATGCCCTTAGATG- ${ }^{\prime}$, and reverse, 5'-AGCTTATGACCCGCACTTAC-3'.

\section{Statistical Analysis}

All experiments were repeated at least three times, and all data are presented as the means \pm SEM. Statistical calculations were analyzed by GraphPad Prism version 5 (GraphPad Software, San Diego, CA). The statistical significance was assessed by an unpaired two-tailed Student's $t$-test. $P<0.05$ was considered statistically significant.

\section{Results}

GGPPS Maintains High Expression during Fetal Lung Development

To evaluate the potential involvement of GGPPS in fetal lung development, we first measured its expression in mice fetal lungs. GGPPS was mainly expressed in the epithelium and maintained expression throughout the fetal lung development period, which implied GGPPS most likely played an extended role during fetal lung development (Figure 1, A-D). Compared with E12.5, GGPPS protein (Figure 1, B and C) and mRNA (Figure 1D) also showed a striking increase since E14.5 (the initiation time of fetal lung branching morphogenesis), which indicated GGPPS might participate in the lung branching events. To further elucidate the physiologic function of GGPPS in fetal lung development, we generated the lung epithelium-specific Ggps 1 knockout mice by crossing the Ggps $I^{\text {floxffox }}$ mice with $S P C$-rtTA mice and TetO-Cre mice. In these lung epithelium-specific Ggpsl knockout mice (SPC-rtTA/ $\left.\mathrm{Tet} O-\mathrm{Cre} / \mathrm{Ggps} 1^{f l o x} / f l o x\right)$, the tet-responsive Cre transgene ( $\mathrm{Tet} O-\mathrm{Cre}$ ) expression was induced in airway epithelial by specific reverse tetracycline transactivator ( $r t T A)$ expression under the control of a 3.7-kb human surfactant pulmonaryassociated protein $\mathrm{C}(S P C)$ promoter in combination with the inducing agent Dox. ${ }^{24}$ The gene targeting and mating strategies are shown in Figure 1E. Ablation of Ggpsl in fetal lung epithelium was achieved by giving the pregnant mothers Dox-containing water, which was initiated from 
A
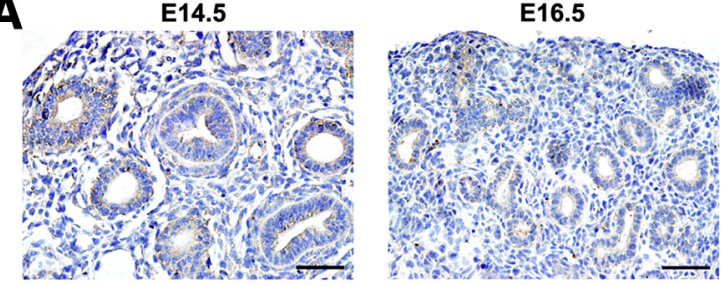

E18.5

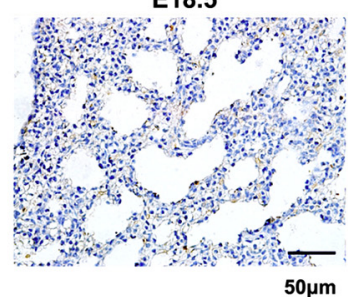

B

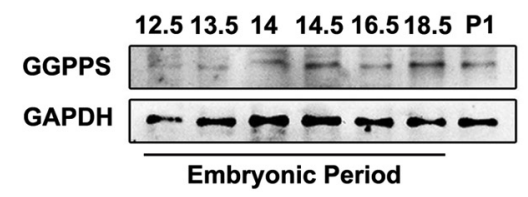

D

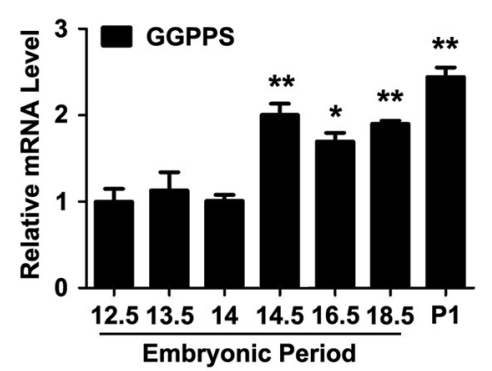

$\mathbf{F}$

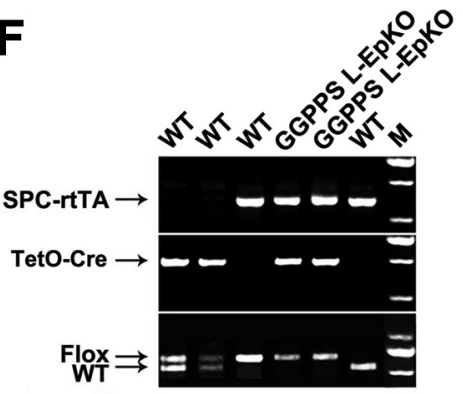

G

\section{C}

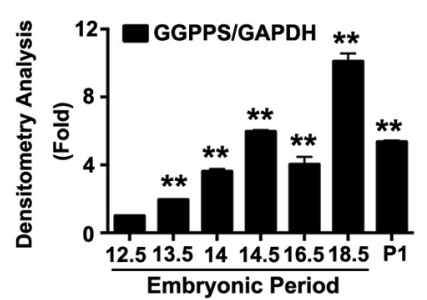

E

SPC-rtTA

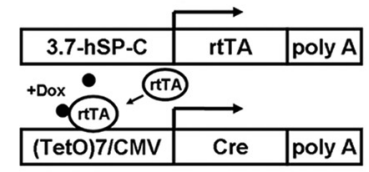

Teto-Cre

Ggps $1^{10 x / f l o x}$

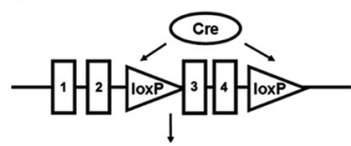

Ggps 1-
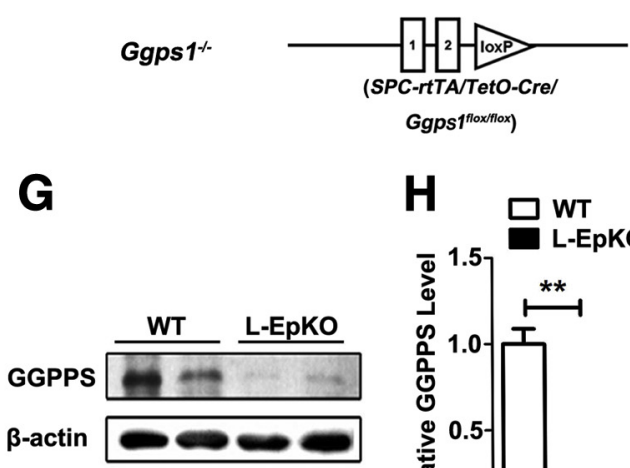

E7.5 or E16.5, and was maintained to the experimental end point. Mice genotypes were validated by tail DNA (Figure 1F). Because fetal lung developments in Ggpsl floxflox,$S P C$-rtTA/ Ggps $1^{\text {flox/flox }}$ and TetO-Cre/Ggps $1^{\text {flox/flox }}$ mice had no difference, we used these normal mice as controls, which were referred to as WT, and the lung epithelium-specific Ggps1 knockout mice hereafter are referred to as Ggps1 L-EpKO. Knockout efficiency of Ggpsl L-EpKO mice was verified at the protein and mRNA levels by Western blot analysis and real-time PCR (Figure 1, G and $\mathrm{H}$ ).

\section{Ablation of Ggps1 in Lung Epithelium Results in NRDS-Like Disease at P1}

The Ggps1 L-EpKO mice died at P1 with observably gasping, cyanosis, and respiratory failure (Figure 2A), and the lungs exhibited pneumonectasis at the terminal alveolus,

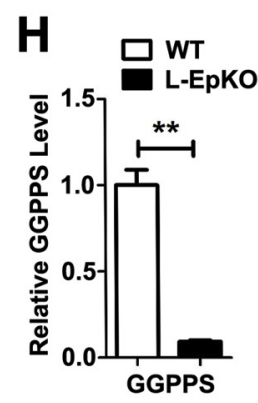

Figure 1 GGPPS maintains expression during fetal lung development and the construction of inducible knockout system in Ggps1 L-EpKO mice is shown. A: GGPPS expression and localization were detected by immunohistochemistry in $\mathrm{C} 57 \mathrm{BL} / 6 \mathrm{~J}$ mouse lungs at E14.5, E16.5, and E18.5. B-D: GGPPS expression was measured by Western blot analysis (B), quantified by densitometry analysis (C), and real-time PCR (D) during fetal lung development, including E12.5, E13.5, E14, E14.5, E16.5, E18.5, and P1. The quantitation of GGPPS expression (C) and relative GGPPS mRNA level (D) at E12.5 were used as comparison. E: Schematic diagram of the targeting construct for doxycyclineinduced lung epithelial-specific ablation of Ggps1 mouse model. F: PCR results of genotyping from tail biopsies of WT and Ggps1 L-EpKO mice. From left to right: TetO-Cre/Ggps1 flox/+ , TetO-Cre/ Ggps $1^{\text {flox/+ }}$, SPC-rtTA/Ggps $1^{\text {flox/flox }}$, SPC-rtTA/TetOCre/Ggps1 $1^{\text {flox/flox }}$, SPC-rtTA/Tet0-Cre/Ggps $1^{\text {flox/flox }}$, and $S P C-r t T A / G g p s 1^{+/+} . \mathbf{G}$ and $\mathbf{H :}$ Ggps1 knockout efficiency was validated by Western blot analysis (G) and real-time PCR (H) in WT and Ggps1 L-EpKO mouse lungs. Data are expressed as means \pm SEM. $n \geq 5$ (A-D); $n=5$ (G and $\mathbf{H})$. ${ }^{*} P<0.05$ and ${ }^{* *} P<0.01$. Cre, Cyclization recombinase; $\mathrm{E}$, embryonic day; flox, loxP-flanked; GAPDH, glyceraldehyde-3-phosphate dehydrogenase; GGPPS, geranylgeranyl diphosphate synthase; L-EpKO, lung epithelium-specific Ggps1 knockout; loxP, locus of $\mathrm{X}$-over $\mathrm{P} 1$; $\mathrm{M}$, marker; $\mathrm{P}$, postnatal day; rtTA, reverse tetracycline-controlled transactivator; SPC, surfactant pulmonary-associated protein $\mathrm{C}$, promoter; Tet0, tetracycline-responsive promoter; WT, wildtype. suggesting a compensatory process after birth (Figure 2B). Phenotypes in the Ggps 1 L-EpKO mice were reported to be the clinical manifestation of NRDS. ${ }^{25}$ In addition, mice displayed similar body weight (Figure 2C), whereas the lung weight was significantly lower in Ggps 1 L-EpKO mice (Figure 2D). Moreover, H\&E staining of Ggps1 L-EpKO mice lungs showed dilated sacs with thin walls, and some sacs exhibited collapsed alveoli structure, indicating the increased surface tension (Figure 2E). At the other area, the terminal sacs failed to inflate with air during postnatal breathing, and fewer expanded sacs in the central area were observed, both of which implied pulmonary atelectasis and dysplasia (Figure 2E). Both of the pneumonectasis and expanded alveolus appeared eosin-stained amorphous (hyaline membrane) (Figure 2E). Furthermore, NRDS-like diseases were caused by insufficient surfactant production, which is comprised of phospholipids and proteins. $^{26}$ 
A

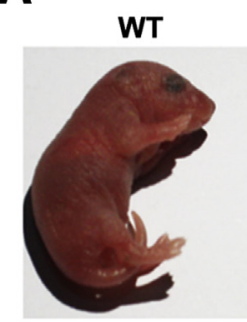

B

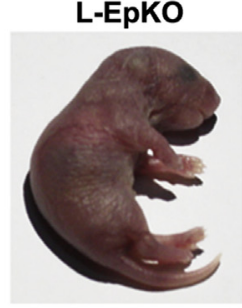

WT

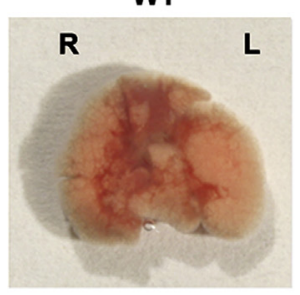

L-EpKO

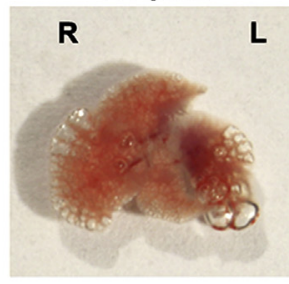

C

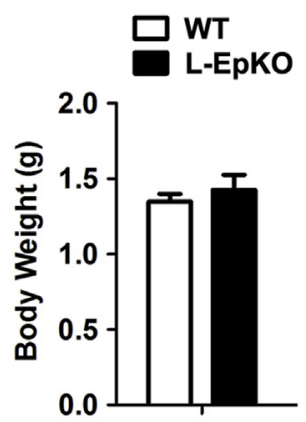

D

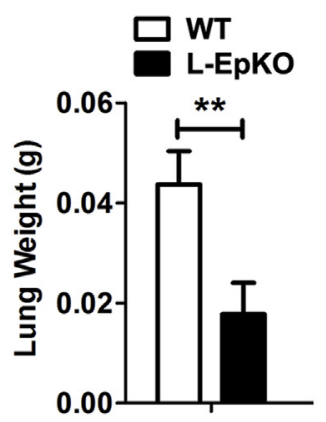

E

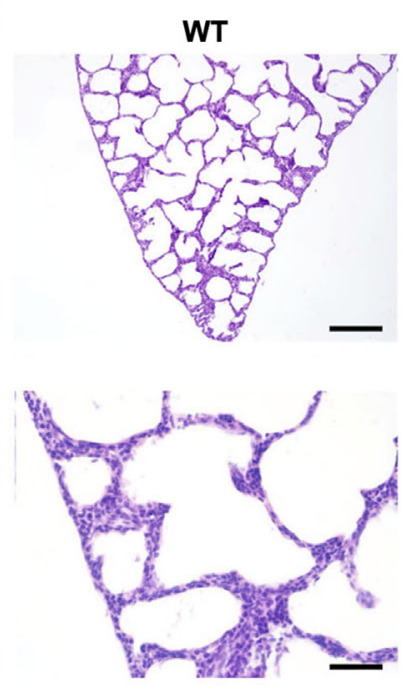

G
L-EpKO
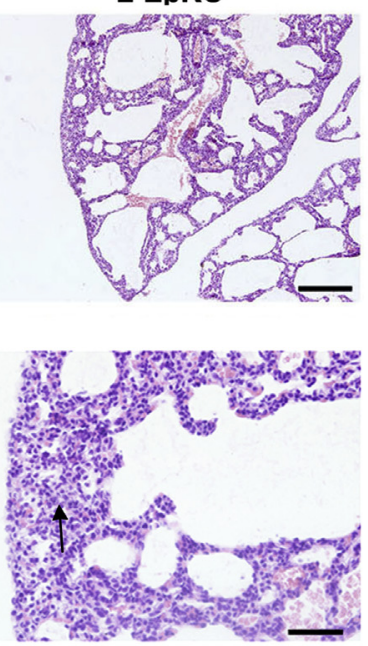

L-EpKO

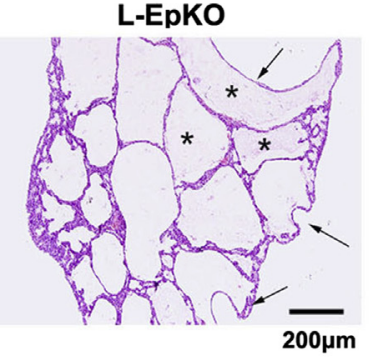

\section{F}

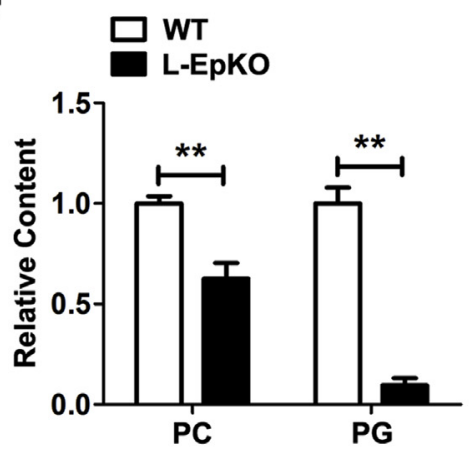

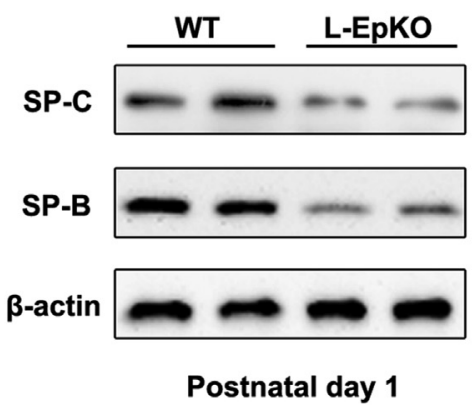

H

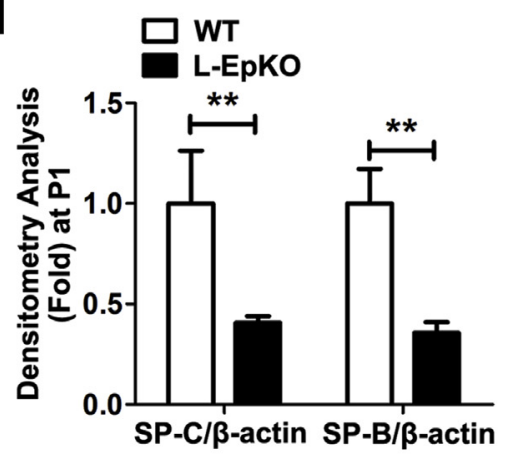

Figure 2 Lung epithelial-specific knockout of GGPS1 causes neonatal respiratory distress syndrome-like disease at P1. A: Ggps1 L-EpK0 neonates display cyanotic phenotype. B: Autopsy observation shows a compensatory expansion and pneumonectasis at terminal alveolus in Ggps1 L-EpK0 mouse lungs at P1. C and D: Body weight (C) and lung weight (D) were weighed in WT and Ggps1 L-EpKO mouse neonates. E: Histologic analysis of neonatal lungs in WT and Ggps1 L-EpK0 mice by hematoxylin and eosin staining. Ggps1 L-EpKO mouse lungs display dilated sacs (right panel) with/without thin walls with collapsed alveoli structure (right panel, arrows), and alveolus appears eosin-stained amorphous (hyaline membrane) (right panel, asterisks). Some of the terminal sacs show failure of air inflation with expanded but fewer sacs in the central area (middle panel, arrow). F: PC and PG contents in neonatal lungs were measured by the mass spectrometry approach, and results are shown as relative PC and PG content in neonatal WT and Ggps1 L-EpKO mice lungs. G and H: Lung tissue SP-B and SP-C were evaluated by Western blot analysis (G) in neonatal WT and Ggps1 L-EpKO mouse lungs, which is quantified by densitometry analysis (H). Data are expressed as means \pm SEM. $n=7(\mathbf{A}-\mathbf{E}) ; n=6(\mathbf{F}-\mathbf{H}) .{ }^{*} P<0.01$. GGPPS, geranylgeranyl diphosphate synthase; L, left; L-EpK0, lung epithelium-specific GGPS1 knockout; $\mathrm{P}$, postnatal day; $\mathrm{PC}$, phosphotidyl choline; $\mathrm{PG}$, phosphatidylglycerol; $\mathrm{R}$, right; SP-B, surfactant protein $\mathrm{B}$; SP-C, surfactant protein C; WT, wild-type.

Because PC and PG are most abundant in lipid portions and SP-B and SP-C are important in forming and stabilizing surfactant layers. ${ }^{26} \mathrm{PC}$ and $\mathrm{PG}$ contents and expression of SP-B and SP-C in neonatal lungs were measured in WT and Ggps 1 L-EpKO mice. Both of the PC and PG contents were largely reduced in Ggpsl L-EpKO mice lungs (Figure 2F).
Furthermore, SP-B and SP-C protein levels also turned out to be declining (Figure 2, G and $\mathrm{H}$ ). These findings interpreted the increased surface tension in Ggpsl L-EpKO mice lungs and demonstrated that Ggps 1 deficiency in lung epithelium caused severe hypoplasia in lung. In addition, we also conducted different periods of Dox induction in 
pregnant mice, either in an induction from E7.5 to midgestation (E17.5 or E18.5) or a continuous Dox administration. Neonatal WT mice exhibited the same lung developments, while the neonatal Ggps 1 L-EpKO mice showed the NRDS-like phenotypes, which implied rtTA toxicity didn't account for the hypoplasia phenotypes in Ggps1 L-EpKO mice as reported earlier. ${ }^{27}$ Herein, we concluded that the lung epithelium GGPPS plays an irreplaceable function during fetal lung development.

\section{Ggps1 Deletion Blocks Lung Branching Morphogenesis during the Pseudoglandular Period and Leads to Mice Prenatal Lung Immaturity}

To elucidate the lung malformation in Ggps1 L-EpKO mice during fetal lung development, prenatal lung characteristics at different developmental stages were examined in detail. The branch numbers were counted in WT and Ggpsl LEpKO mice lungs. No apparent difference was found in early branching morphogenesis (E12.5) (Figure 3A), whereas the macroscopic observations showed expansive distal pre-acini/ alveolus in Ggps1 L-EpKO mice lungs at E16.5 and E18.5 (Figure 3A). Moreover, through H\&E staining, the lumens/ pre-acini/alveolus of Ggpsl L-EpKO mice lungs showed expanded phenotype, and some exfoliative cells appeared in the lumen at E14.5, E16.5, and E18.5 (Figure 3B). Meanwhile, the morphologic abnormalities of Ggpsl L-EpKO mice lungs at E16.5 and E18.5 became obvious, the pre-acini/ alveolus exhibited profound pathologic changes (Figure 3B). Dilated and fewer pre-acini/alveolus and thickened mesenchyme were also observed, which were relevant to Ggps $1 \mathrm{~L}-$ EpKO mice lungs as described in the previous paragraph at P1. At the same time, by staining lung epithelium with the specific marker SP-C, the exfoliative cells were found to be epithelial derived (Figure 3C). Glycogen was reported to be accumulated in epithelium from lung bud formation to the pseudoglandular period, and then they were converted to phospholipids and mobilized to lamellar bodies during lung epithelial cell growth, differentiation, and maturation. ${ }^{28}$ Therefore, glycogen accumulation reflects an immaturity of peripheral lung epithelial cells after E17.5. The cytoplasm positive for glycogen staining was much more enriched in Ggps1 L-EpKO mice lungs than in WT lungs at E18.5, which indicated the developmental immaturity after Ggpsl deletion (Figure 3D). To better address which period accounted for phenotypes we observed in Ggpsl L-EpKO mice at E14.5, E16.5, E18.5 and P1, we delayed the Dox induction time to E16.5 (Figure 3E). All of the neonatal E16.5 induced Ggps1 L-EpKO mice (E16.5 L-EpKO) survived, and their lungs showed no obvious difference except for some exfoliative cytes in the lumen at P1 compared with WT mice (Figure 3F). Taken together, our findings suggested that Ggps 1 deficiency in lung epithelium led to prenatal lung malformation and immaturity, which resulted from the defective lung branching morphogenesis, especially the branching morphogenesis during the pseudoglandular period.

\section{Decreased Proliferation and Differentiation and Increased Apoptosis Contribute to the Lung Malformation in Ggps1 L-EpK0 Mice}

Ggpsl L-EpKO mice exhibited a dilated and fewer preacinar/ alveolus and thickened mesenchyme phenotype, which might result from the alteration of cell proliferation and apoptosis behavior. The cell proliferation was measured by counting $\mathrm{Ki}-67^{+}$cells in 10 lung epithelial cells per lumen/pre-acini. The number of Ki- $67^{+}$lung epithelial cells was significantly reduced in Ggps 1 L-EpKO mice at E14.5 and E16.5 compared with those in WT mice lungs (Figure 4, A, B, and D). In addition, we could not distinguish epithelium from mesenchyme with histologic methods at E18.5, but alveolus septa displayed an abnormal active proliferation activity, implying a delayed development accompanied with failure entering lung differentiation in Ggpsl L-EpKO mice lungs (Figure 4C), and this striking proliferation activity in fetal lung mesenchyme may account for the thickened mesenchyme phenotype at P1. We further measured the apoptosis behavior by the TUNEL assay. The number of apoptotic cells in 100 lumens was increased at E14.5, E16.5, and E18.5 versus WT, implying a more active apoptosis behavior in Ggpsl L-EpKO mice lung epithelium (Figure 4, E-H). Therefore, the malformation of expanded lumens at E14.5 and E16.5 in Ggpsl L-EpKO mice lungs might result from reduced proliferation and increased apoptosis in epithelium.

We also checked the lung differentiation progress by detecting the expression of differentiated markers. Fetal lung proximal and distal epithelial will enter into differentiation that is accompanied by unique molecular marker expression from E14.5, despite intensive proliferation activity. Proximal airway epithelial cells differentiate into ciliated columnar cells with expression of unique molecular markers, including FoxJ1. The transitional region of airways is lined with differentiated Clara cells, which can be distinguished by CCSP (or CC10) expression. Moreover, distal lung epithelial cells express surfactant proteins and (AQP5), and they have the potential to become type I and type II pneumocytes after birth. Thus, we detected these genes by real-time PCR. The proximal epithelium marker FoxJ1 were unaffected by Ggps1 deletion at E14.5, E16.5, and E18.5, whereas the distal epithelium marker SP-B was markedly reduced. At E18.5, the expressions of CCSP, SP-B, and AQP5 were all largely decreased in Ggps 1 L-EpKO mice as well (Figure 4I). The relative expression of FoxJ1, CCSP, SP-B, and AQP5 at E14.5, E16.5, and E18.5 are shown in Supplemental Figure S1.

Taken together, Ggps1 deficiency in epithelium reduced epithelial proliferation and altered epithelial differentiation and increased epithelial apoptosis behavior, thus contributing to the lung malformation, immaturity, and malfunction as observed at P1 in Ggps1 L-EpKO mice. 
A

E12.5

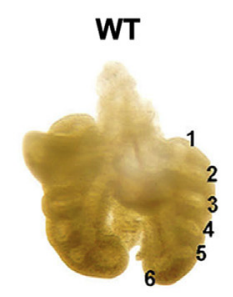

WT

E16.5
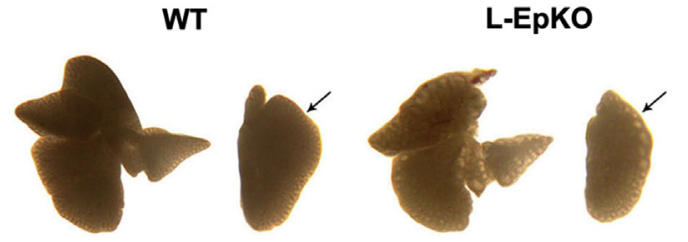

E18.5
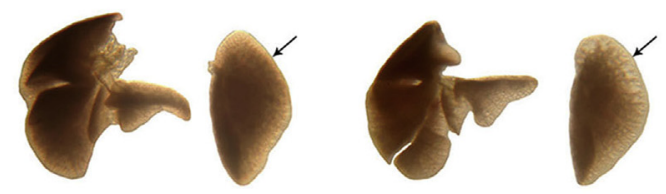

C
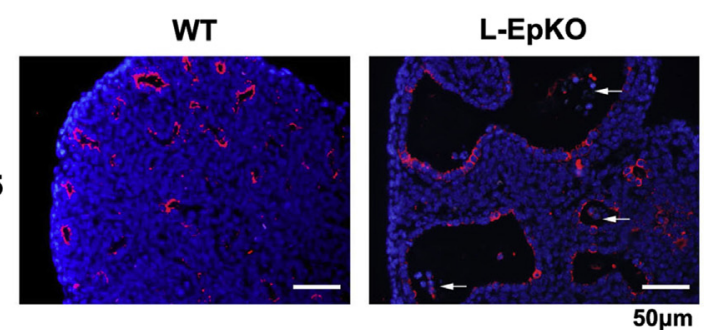

E

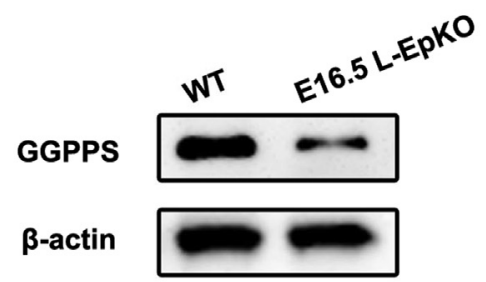

Postnatal day 1
B WT
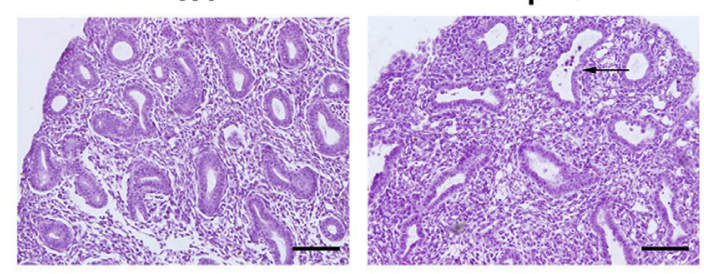

E16.5
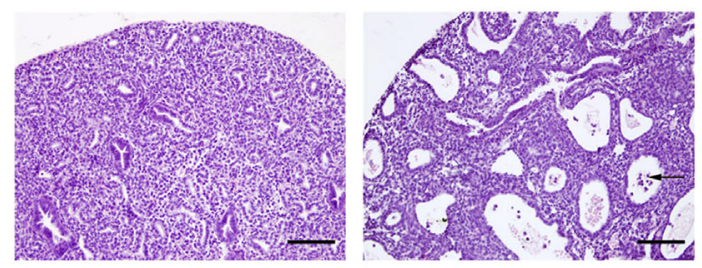

E18.5
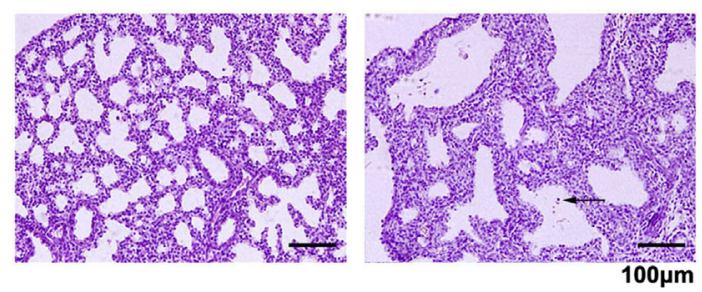

D

WT
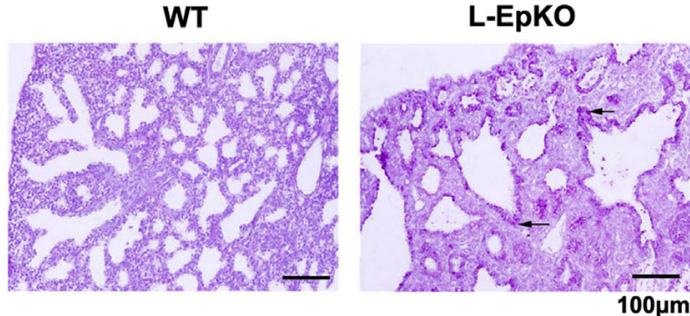

F

WT

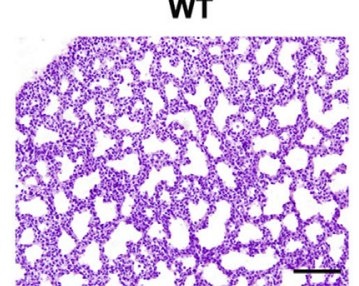

E16.5 L-EpKO

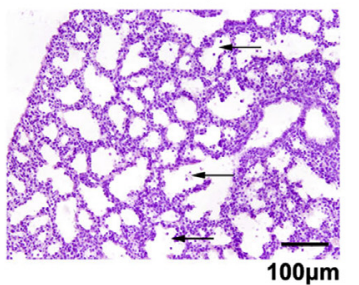

Figure 3 Ggps1 deletion blocks lung branching morphogenesis and leads to prenatal lung immaturity. A: Autopsy observation shows gross lung morphology in WT and Ggps1 L-EpKO mice during lung development. The branching numbers were manually counted, and no difference was found in WT compared with Ggps1 L-EpKO mouse lungs at E12.5. Moreover, distal pre-acini/alveolus show compensatory expansion and pneumonectasis at terminal alveolus in Ggps1 L-EpK0 mouse lungs at E16.5 and E18.5 (arrows). B: Histologic analysis of embryonic lungs shows expanded distal lumens/pre-acini/alveolus in Ggps1 L-EpK0 mice at E14.5, E16.5, and E18.5 with exfoliative cytes in the lumen (arrows). C: Expression of lung epithelial cells marker SP-C in E16.5 mouse lungs. The exfoliative cytes in the lumen of Ggps1 L-EpKO mouse lungs were epithelium-derived (arrows). D: Periodic acid-Schiff staining was performed in WT and Ggps1 L-EpKO mouse lungs at E18.5. Excessive accumulation of glycogen (arrows) in the cytoplasm in Ggps1 knockout mice reveals lung developmental immaturity. E: The knockout efficiency of E16.5-induced Ggps1 L-EpK0 mice were measured by Western blot analysis at P1. F: Compared with WT, histologic analysis of E16.5-induced Ggps1 L-EpK0 mice lungs show no obvious difference except for some exfoliative cytes in the lumen (arrows) at P1. $n=5$. E, embryonic day; GGPPS, geranylgeranyl diphosphate synthase; L-EpK0, lung epithelium-specific Ggps1 knockout; P, postnatal day; SP-C, surfactant protein C; WT, wild-type.

Ggps1 Deficiency Inhibits the Lung Branching Morphogenesis Likely by Interfering with FGF10 Signaling

Lung branching morphogenesis is a well-orchestrated process, which is tightly controlled by the interplay of several key factors, including FGF10, Sprouty2, Shh, and BMP4..$^{5}$ FGF10 is synthesized in lung mesenchyme and functions to promote epithelial Sprouty2, Shh, and BMP4 expression in a paracrine manner. Then, the expression of Sprouty2, Shh, and BMP4 in turn suppresses the FGF10 signaling on distal epithelium proliferation and differentiation. Thus, the 
A
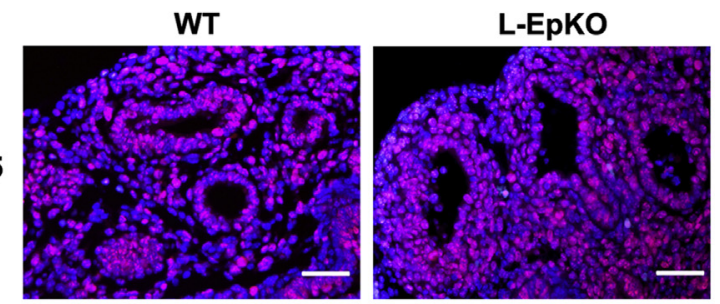

$50 \mu \mathrm{m}$

C

WT
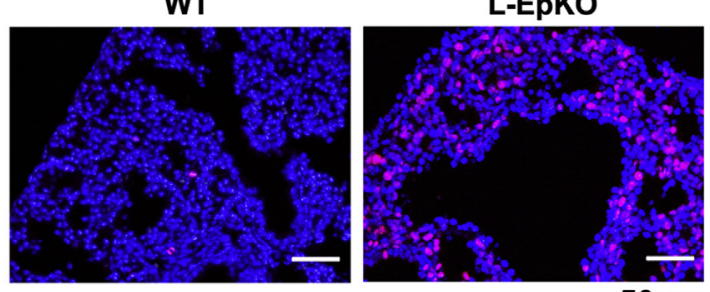

$50 \mu \mathrm{m}$

E

WT
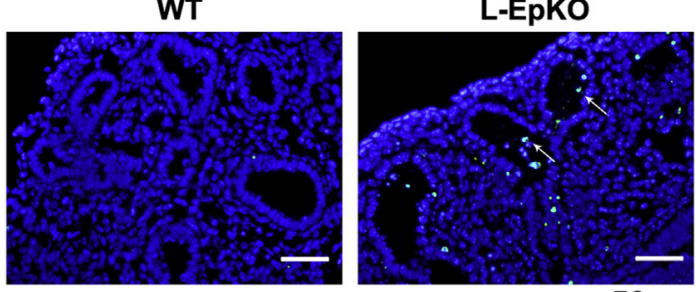

$50 \mu \mathrm{m}$

G
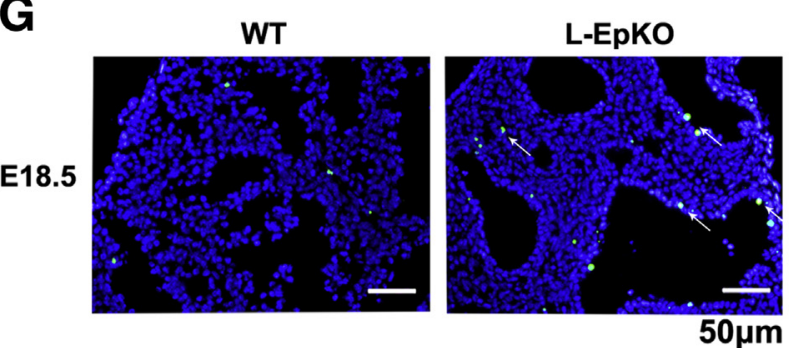

I

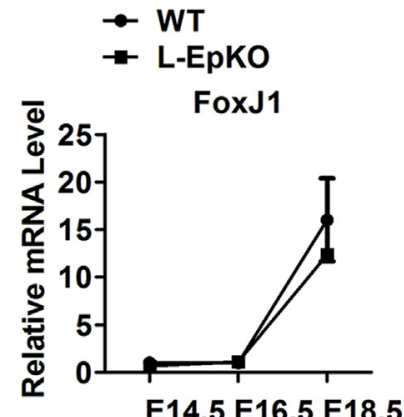

B
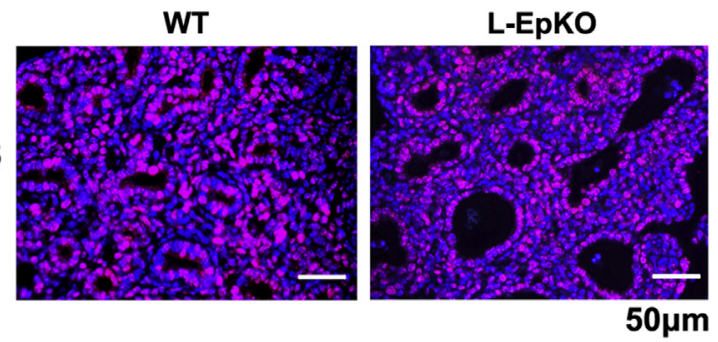

D

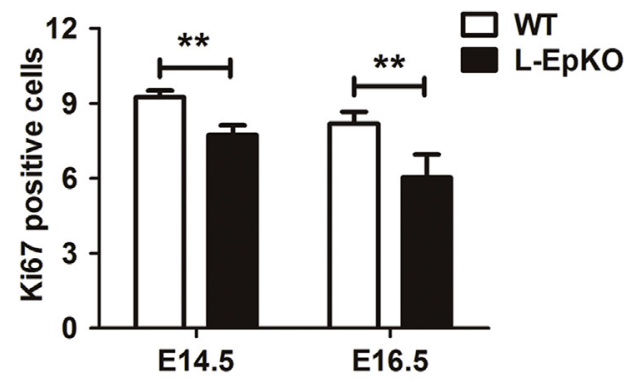

$F$

WT
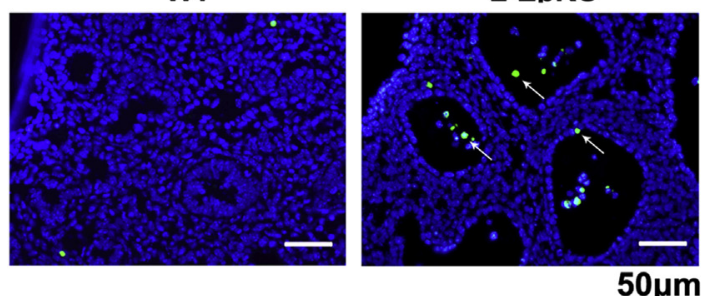

H
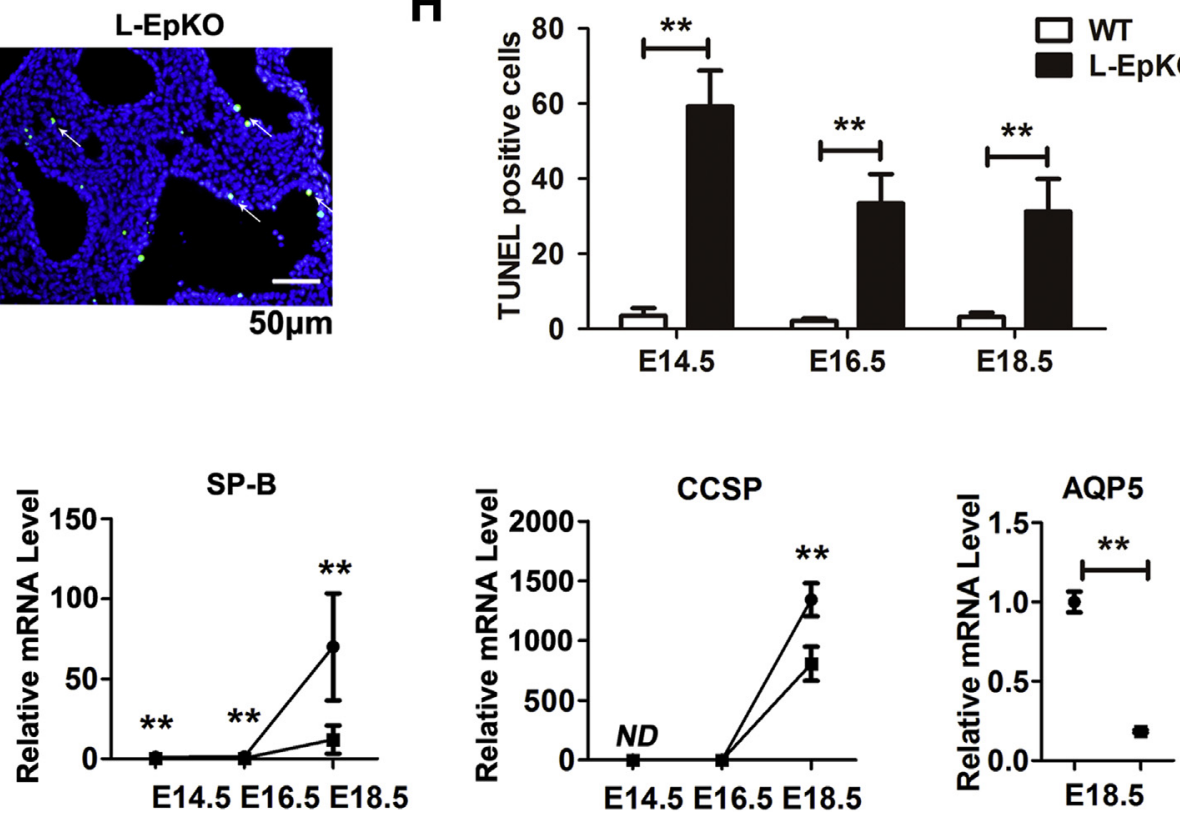

Figure 4 Deletion of Ggps1 in lung epithelial cells results in decreased proliferation activity, increased apoptosis behavior, and decreased differentiation marker gene expression. A-D: Cell proliferation was evaluated by Ki-67 staining (red) in WT and L-EpK0 mouse lungs at E14.5 (A), E16.5 (B), and E18.5 (C). Nuclei were stained with DAPI (blue). The quantification represents Ki-67 $7^{+}$cells in 10 epithelial per lumen/preacinar (D). E-H: Apoptotic cells in WT and Ggps1 L-EpK0 mouse lungs were detected by TUNEL (green, arrows) at E14.5 (E), E16.5 (F), and E18.5 (G). Nuclei were stained with DAPI (blue). The quantification represents the number of TUNEL ${ }^{+}$cells in 100 lumens (H). I: Expression of differentiation marker genes for lung epithelial cells in E14.5, E16.5, and E18.5 embryonic lungs (relative expression of these markers at E14.5, E16.5, and E18.5, respectively, are shown in Supplemental Figure S1, A-C). Data are expressed as means \pm SEM. $n=5(\mathbf{A}-\mathrm{I}) .{ }^{* *} P<0.01$. AQP5, aquaporin-5; CCSP, Clara cell-specific protein; E, embryonic day; FoxJ1, forkhead box protein J1; L-EpKO, lung epithelium-specific Ggps1 knockout; ND, nondetected; SP-B, surfactant protein B; TUNEL, terminal deoxynucleotidyl transferase-mediated dUTP nick-end labeling; WT, wild-type. 
$A \rightarrow$ WT

$\rightarrow$ L-EpKO

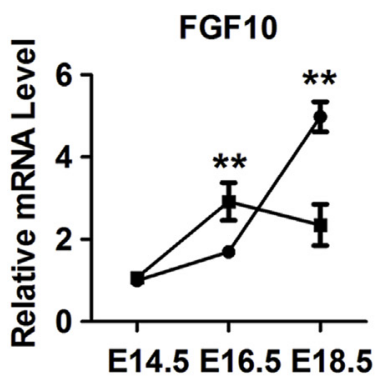

Shh

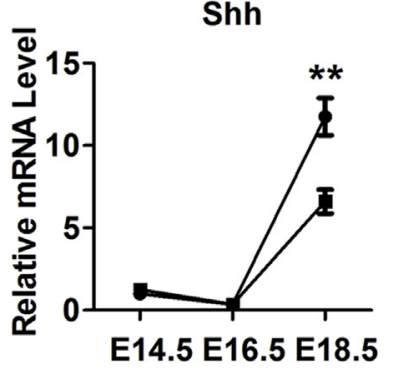

D

WT

E14

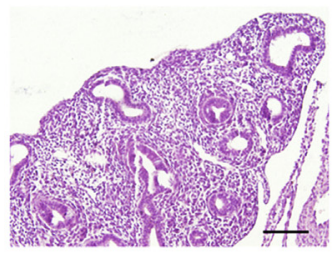

E14

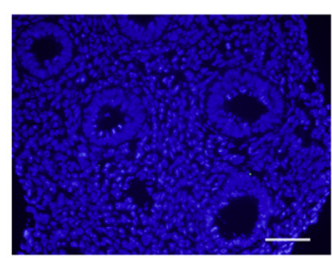

B

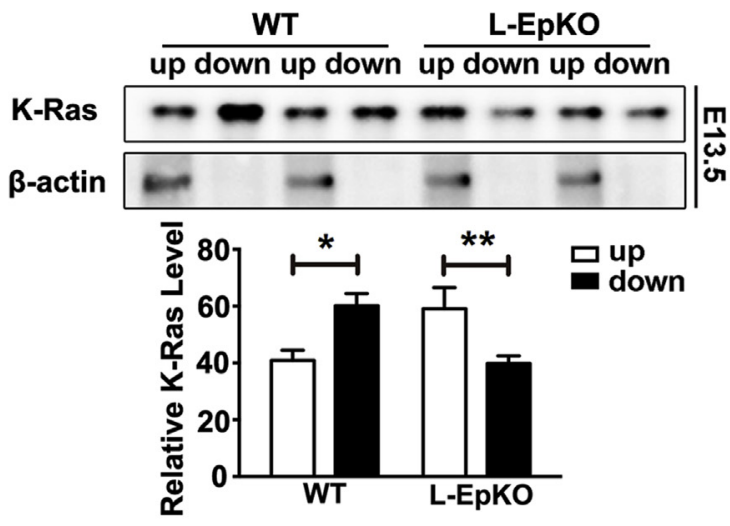

C
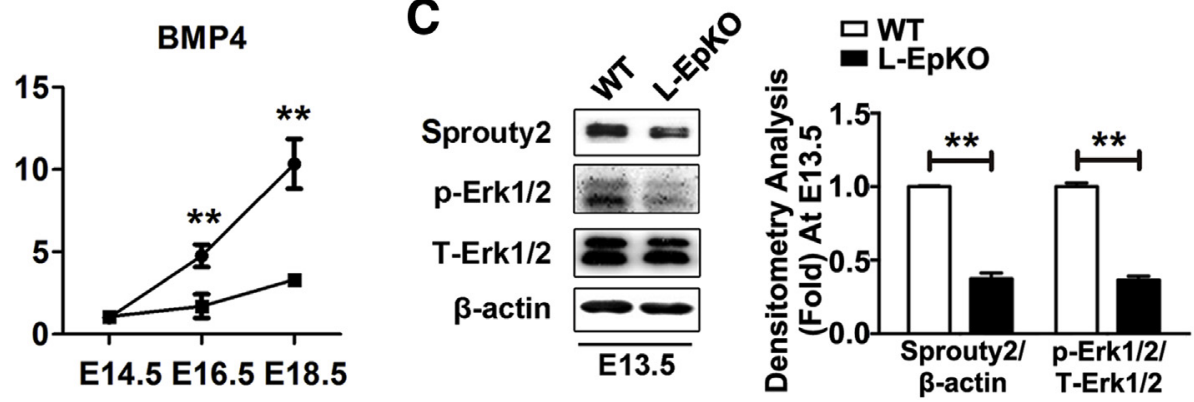

E
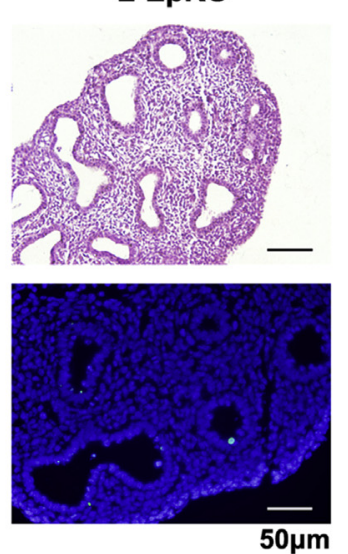

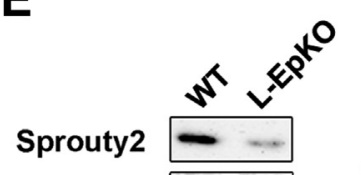

p-Erk1/2

T-Erk1/2

p-Akt

T-Akt

$\beta$-actin
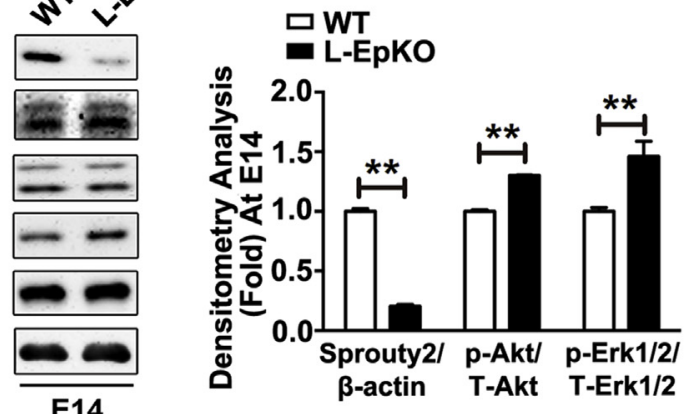

Figure 5 GGPPS-controlled K-Ras prenylation and Erk1/2/MAPK signaling mediates FGF signaling on lung branching morphogenesis. A: Key factors such as FGF10, Shh, BMP4, and Sprouty2, involved in lung branching morphogenesis, were detected in the lung epithelial cells in E14.5, E16.5, and E18.5 embryonic lungs (relative expression of these factors at E14.5, E16.5, and E18.5, respectively, are shown in Supplemental Figure S2, A-C). B: Subcellular fractionation in the lung was performed with the Triton X-114 partition method. The aqueous upper phase contained the water-soluble small GTPases and the organic lower phase contained the lipid-soluble small GTPases. K-Ras prenylation was evaluated in the lung of E13.5 WT and Ggps1 L-EpK0 mice. C: Sprouty2 and phosphorylation of Erk1/2 were assessed in E13.5 lungs of WT and Ggps1 L-EpK0 mice. D: Lung morphology and apoptotic epithelial cells were analyzed with hematoxylin and eosin staining and TUNEL assay. Nuclei were stained with DAPI (blue). E: Sprouty2, phosphorylation of Erk1/2 and Akt were assessed in E14 lungs of WT and Ggps1 L-EpK0 mice by Western blot analysis. Data are expressed as means \pm SEM. $n=5(\mathbf{A}$ and $\mathbf{C}-\mathbf{E}) ; n=6(\mathbf{B})$. ${ }^{*} P<0.05,{ }^{* *} P<0.01$. BMP4, bone morphogenetic protein 4; down, lipid-soluble small GTPases; E, embryonic day; FGF10, fibroblast growth factor 10; GGPPS, geranylgeranyl diphosphate synthase; L-EpKO, lung epithelium-specific Ggps1 knockout; MAPK, mitogen-activated protein kinase; p-Akt, phosphorylated Akt; p-Erk1/2, phosphorylated extracellular signal-related kinase 1/2; Shh, sonic hedgehog; T-Akt, total Akt; T-Erk1/2, total Erk1/2; TUNEL, terminal deoxynucleotidyl transferase-mediated dUTP nick-end labeling; up, water-soluble small GTPases; WT, wild-type.

proper interaction between FGF10 signaling and Sprouty2, Shh, and BMP4 is necessary for lung branching morphogenesis. ${ }^{4}$ To uncover the mechanism of branching defects in Ggps1 L-EpKO mice lungs, we evaluated the expression of FGF10, Sprouty2, Shh, and BMP4 during the branching morphogenesis. The real-time PCR showed the expression of FGF10, Shh, and BMP4 had no obvious difference, whereas Sprouty2 was decreased in Ggps1 L-EpKO mice lungs at E14.5 (Figure 5A). Then at E16.5, FGF10 experienced a striking increase, which might result from the reduction of Sprouty2 at E14.5 and E16.5 and BMP4 at E16.5 (Figure 5A). At the end of branching morphogenesis 
(at E18.5), FGF10, Shh, Sprouty2, and BMP4 were all markedly reduced (Figure 5A), suggesting lung development was completely blunted in Ggps 1 L-EpKO mice lungs. Taken together, our data indicated that knockout of Ggpsl in lung epithelium inhibited the branching events, likely through interfering with the interplay between FGF10 signaling and Sprouty2, Shh, and BMP4. The relative expression of FGF10, Sprouty2, Shh, and BMP4 at E14.5, E16.5, and E18.5 are shown in Supplemental Figure S2.

\section{GGPPS Mediates FGF Signaling on Lung Branching Morphogenesis through Controlling K-Ras Prenylation and Erk1/2/MAPK Signaling}

To extend our knowledge on how GGPPS affected FGF10 regulation on branching morphogenesis, we examined K-Ras/ MAPK and phosphoinositide 3-kinase (PI3K)/Akt signaling pathways that were reported to mediate the FGF10 signals in lung epithelium. Consistent with our previous study, the KRas membrane association was largely blocked after Ggps 1 deletion in lung epithelium (Figure $5 \mathrm{~B}$ ). ${ }^{20}$ This was further confirmed by measuring the activation of Erk1/2 and downstream Sprouty2, both of which were reduced after Ggps 1 deletion at E13.5 (Figure 5C). These results indicated that ablation of Ggps1 in lung epithelium decreased the K-Ras prenylation, thus abrogated the K-Ras/MAPK signaling and Sprouty2 expression. Furthermore, our earlier detections revealed a remarkable immaturity in lungs of Ggps1 L-EpKO mice versus WT at E14.5 (Figure 3B). As we compared the morphology and distal epithelial cell behavior at E14, no discriminable difference was found in Ggps 1 L-EpKO mice lungs compared with WT (Figure 5D). Therefore, we focused on the alteration of signals during the transit point at E14. Ggps1 L-EpKO mouse lungs showed markedly reduced expression of Sprouty2 at E14, together with a slightly increased intracellular MAPK signaling (Figure 5E). This is consistent with previously reported data that Sprouty2 is a negative regulator of Erk1/2/MAPK signaling. ${ }^{10}$ However, the interfered (impaired and activated) K-Ras/MAPK signaling led to the reduced Sprouty2 expression in Ggps1 L-EpKO mice, which in turn contributed to the lung immaturity. Herein, we observed a blunted interplay between Sprouty2 and K-Ras/ MAPK in Ggps I L-EpKO mice; and activation of which was required for lung branching morphogenesis. Intriguingly, activation of PI3k/Akt signaling was also observed, which might be caused by the elevation of FGF10 signaling, resulting from the reduced suppression by Sprouty2 (Figure 5A). Taken together, GGPPS is essential for fetal lung branching morphogenesis, which might be through mediating FGF10 signaling by controlling K-Ras prenylation and subsequent Erk1/2/MAPK activation.

\section{Discussion}

Lung branching morphogenesis is a well-orchestrated process that is tightly controlled by complex signaling pathways, including FGF10 signaling. ${ }^{5}$ Alteration of this pathway during the fetal lung development suppresses the cascade of K-Ras/MAPK and Sprouty2 expression, thus resulting in prenatal lung malformation and neonatal respiratory insufficiency or respiratory failure. ${ }^{29}$ In this research, we demonstrated a critical role of lung epithelium GGPPS in regulating lung branching morphogenesis and proposed a potential mechanism that K-Ras prenylation might account for this effect. Specific deletion of Ggps1 in lung epithelium led to severe respiratory failure and NRSD-like disease that resulted from the failure of lung branching morphogenesis. The potential mechanism was Ggps 1 deficiency inhibited KRas/MAPK signaling, which in turn abrogated FGF10 regulation on fetal lung morphogenesis. Therefore, GGPPS plays an essential role in maintaining the fetal lung branching morphogenesis possibly through controlling KRas prenylation.

FGF10 signaling is a principal pathway in regulating the development of various organs, including lung, mammary gland, kidney, and prostate. ${ }^{30}$ It maintains expression throughout embryonic lung development ${ }^{31,32}$ and controls fetal lung branching morphogenesis and the bud outgrowth. ${ }^{31}$ Previous studies indicated that specific deletion of FGF10 in parabronchial smooth muscle resulted in severe hypoplasia and dilation of the distal airways. ${ }^{33}$ These were caused by the reduced proliferation behavior because of the interference of downstream K-Ras/MAPK signaling and the abnormal interplay between FGF10 and its negative regulator Sprouty2. ${ }^{34} \mathrm{~A}$ study also revealed the constitutive activated Ras/MAPK signaling prevented the normal airway shape changes in Shh-Cre/Kras ${ }^{L S L-G 12 D}$ transgenic mice. On combining with PD0325901, a highly specific inhibitor of MAPK kinase1/2, the normal airway formation was restored at E11.5. ${ }^{11}$ However, the subsequent effect on branching morphogenesis or whether inactivation of K-Ras in fetal lung epithelium would interfere with fetal lung development was not interpreted.

Here, we found GGPPS consistently expressed in lung epithelium throughout the lung development, and the expression of GGPPS was increased from the pseudoglandular period (E14.5) (Figure 1, A-D). Through the specific knockout of Ggps1 in lung epithelium, we proved GGPPS mediated the branching morphogenesis, likely by controlling K-Ras prenylation. In particular, the defective branching events were observed as early as the pseudoglandular period (E14.5), which led to the malformation of the future bronchi tree and subsequently alveolus formation and function, which were evidenced by the enlarged lumens and the decreased differentiation markers at E18.5 (Figure 4I). Thus, our investigation completes the function of K-Ras/MAPK signaling pathway in fetal lung development that is necessary for FGF10 signaling on branching morphogenesis.

In addition, the net effect of Ggps 1 deletion in epithelium also resulted in the abnormal expression of Sprouty2 and damaged its interaction with FGF10 signaling. Sprouty2 is 
expressed in lung epithelium because the bud formation and the expression keeps increasing as the bud grows. ${ }^{35}$ It mediates the FGF10/FGFR2 regulation on branching morphogenesis and the airway epithelial tubular growth. ${ }^{36}$ In our study, we found a reduced expression of Sprouty2 in the lung epithelium of Ggps 1 L-EpKO mice initiated from E13.5 to E18.5 (Figure 5, A, C, and E). Although the decrease of Sprouty2 released the repression of FGF10 signaling and activation of MAPK signaling as we detected at E16.5 (Figure 5A) and E14 (Figure 5E), Ggps 1 deficiency markedly impaired the expression of Sprouty2, thus constantly interfered with the FGF10 signaling on lung branching morphogenesis. Therefore, GGPPS is irreplaceable during fetal lung branching morphogenesis, likely through maintaining the interplay of Sprouty2 and FGF10 by controlling K-Ras prenylation and Erk1/2/MAPK signaling.

FGF10 signaling is also reported to modulate endoderm proliferation and bud outgrowth in fetal lung by binding to FGFR2. ${ }^{31}$ FGF10-deficient mice and FGFR2-null mice died as infants with lung agenesis, ${ }^{37,38}$ and $\mathrm{FGFIO}^{+/-} / \mathrm{Mlclv}$ $\mathrm{LacZ}^{+/-}$mice showed the absence of the lung accessory lobe. ${ }^{33}$ Although we interrupted the FGF10/FGFR2/ Sprouty2 network, Ggps1 L-EpKO mice displayed the entire lung organ (five lung lobes) (Figures $2 \mathrm{~B}$ and $3 \mathrm{~A}$ ), which indicated a normal lung bud formation, and influenced the lung branching morphogenesis (Figure 3B). These data suggest that the K-Ras/MAPK might participate in branching morphogenesis rather than initiating primary bud formation by mediating the FGF10 signaling. Moreover, emerging evidence suggested the importance of PI3K signaling in regulating branch initiation ${ }^{39}$ and the branching morphogenesis. ${ }^{40} \mathrm{PI} 3 \mathrm{~K}$ responds to the FGF10 signals and exerts on activating Akt, of which the sustained activation defines the branch initiation sites at E11.5. However, the inhibition of PI3K/Akt prevented branch initiation. ${ }^{39}$ Here, we observed activation of Akt signaling in Ggpsl-deficient lungs at E14 (Figure 5E), which might account for the intact bud formation and branch initiation in the situation of Ras/MAPK signaling blockage in Ggps 1 L-EpKO mice. In addition, this elevation of Akt activity might result from the compensatory increase of FGF10 signaling (Figure 5A) or the direct deficiency of Ggpsl as we previously reported. ${ }^{17}$ However, whether the PI3K/Akt network is indeed involved in the lung bud formation and branch initiation remains to be determined.

Besides K-Ras, GGPPS also controlled cellular GGPP to influence prenylation of other $\mathrm{G}$ proteins. ${ }^{12,41}$ For instance, Rac1, a small Rho GTPase, plays a key role in cell polarity and cell migration, which can also be modified by geranylgeranylation. Rac1 was found to activate at the leading edge and is required for branch elongation in mammary epithelium. ${ }^{39}$ Soula Danopoulos et $\mathrm{al}^{42}$ mentioned the involvement of Rac1 in regulating branching morphogenesis by using Rac1 inhibitor in the American Thoracic Society International Conference in 2015. This Rac1 inhibitor, NSC23766, could abrogate the branching morphogenesis by inhibiting the canonical Wnt signaling. ${ }^{42}$ However, because GGPPS is a branch-point enzyme in the mevalonate pathway by catalyzing the synthesis of GGPP from FPP, deletion of Ggps I may also interfere with lipid metabolism and result in PC and PG deficiency, which can cause the NRDS-like disease at P1. Therefore, our research provides one possibility of how GGPPS affects the lung branching morphogenesis; however, the exact mechanism remains to be investigated.

In summary, our results establish a critical role of GGPPS in controlling lung branching morphogenesis. In addition, we propose one potential mechanism: through synthesizing GGPP, GGPPS modulated K-Ras prenylation and Ras/ MAPK signaling, thus mediating the FGF10 regulation on branching morphogenesis. Moreover, Ggpsl deficiency might also cause the activation of PI3K/Akt signaling during the fetal lung development. Thus, our data suggest that GGPPS regulates branching morphogenesis of fetal lung, likely through tilting the balance between Ras/MAPK and PI3K/Akt signaling networks.

\section{Acknowledgments}

W.-J.J., S.J., and C.-J.L. studied the concept and designed the research; W.-J.J., S.J., and Q.-L.T. performed the research and acquired the data; W.-J.J., S.J., and D.S. analyzed and interpreted the data; W.N. provided the reagents; W.-J.J., S.J., Q.-L.T., and C.-J.L. wrote the paper; S.J., Q.-L.T., D.S., B.X., W.N., and C.-J.L. revised the paper. All authors reviewed the results and approved the final version of the manuscript.

\section{Supplemental Data}

Supplemental material for this article can be found at http://dx.doi.org/10.1016/j.ajpath.2016.01.021.

\section{References}

1. Nkadi PO, Merritt TA, Pillers DA: An overview of pulmonary surfactant in the neonate: genetics, metabolism, and the role of surfactant in health and disease. Mol Genet Metab 2009, 97:95-101

2. Ten Have-Opbroek AA: Lung development in the mouse embryo. Exp Lung Res 1991, 17:111-130

3. Chinoy MR: Lung growth and development. Front Biosci 2003, 8: d392-d415

4. Hogan BL, Grindley J, Bellusci S, Dunn NR, Emoto H, Itoh N: Branching morphogenesis of the lung: new models for a classical problem. Cold Spring Harb Symp Quant Biol 1997, 62:249-256

5. Morrisey EE, Hogan BL: Preparing for the first breath: genetic and cellular mechanisms in lung development. Dev Cell 2010, 18:8-23

6. Park WY, Miranda B, Lebeche D, Hashimoto G, Cardoso WV: FGF10 is a chemotactic factor for distal epithelial buds during lung development. Dev Biol 1998, 201:125-134

7. De Moerlooze L, Spencer-Dene B, Revest JM, Hajihosseini M, Rosewell I, Dickson C: An important role for the IIIb isoform of fibroblast growth factor receptor 2 (FGFR2) in mesenchymal-epithelial signalling during mouse organogenesis. Development 2000, 127: 483-492 
8. Cabrita MA, Christofori G: Sprouty proteins, masterminds of receptor tyrosine kinase signaling. Angiogenesis 2008, 11:53-62

9. Bos JL: ras oncogenes in human cancer: a review. Cancer Res 1989, 49:4682-4689

10. Shaw AT, Meissner A, Dowdle JA, Crowley D, Magendantz M, Ouyang C, Parisi T, Rajagopal J, Blank LJ, Bronson RT, Stone JR, Tuveson DA, Jaenisch R, Jacks T: Sprouty-2 regulates oncogenic Kras in lung development and tumorigenesis. Genes Dev 2007, 21: 694-707

11. Tang N, Marshall WF, McMahon M, Metzger RJ, Martin GR: Control of mitotic spindle angle by the RAS-regulated ERK1/2 pathway determines lung tube shape. Science 2011, 333:342-345

12. Wright LP, Philips MR: Thematic review series: lipid posttranslational modifications. CAAX modification and membrane targeting of Ras. J Lipid Res 2006, 47:883-891

13. Wang XX, Ying P, Diao F, Wang Q, Ye D, Jiang C, Shen N, Xu N, Chen WB, Lai SS, Jiang S, Miao XL, Feng J, Tao WW, Zhao NW, Yao B, Xu ZP, Sun HX, Li JM, Sha JH, Huang XX, Shi QH, Tang H, Gao X, Li CJ: Altered protein prenylation in Sertoli cells is associated with adult infertility resulting from childhood mumps infection. J Exp Med 2013, 210:1559-1574

14. Xu N, Guan S, Chen Z, Yu Y, Xie J, Pan FY, Zhao NW, Liu L, Yang ZZ, Gao X, Xu B, Li CJ: The alteration of protein prenylation induces cardiomyocyte hypertrophy through Rheb-mTORC1 signalling and leads to chronic heart failure. J Pathol 2015, 235:672-685

15. Chen WB, Lai SS, Yu C, Liu J, Jiang S, Zhao DD, Ding YT, Li CJ, Xue B: GGPPS deficiency aggravates CCl4-induced liver injury by inducing hepatocyte apoptosis. FEBS Lett 2015, 589:1119-1126

16. Yu DC, Liu J, Chen J, Shao JJ, Shen X, Xia HG, Li CJ, Xue B, Ding YT: GGPPS1 predicts the biological character of hepatocellular carcinoma in patients with cirrhosis. BMC Cancer 2014, 14:248

17. Yu X, Shen N, Zhang ML, Pan FY, Wang C, Jia WP, Liu C, Gao Q, Gao X, Xue B, Li CJ: Egr-1 decreases adipocyte insulin sensitivity by tilting PI3K/Akt and MAPK signal balance in mice. EMBO J 2011, 30: 3754-3765

18. Jiang S, Shen D, Jia WJ, Han X, Shen N, Tao W, Gao X, Xue B, Li CJ: GGPPS-mediated Rab27A geranylgeranylation regulates beta-cell dysfunction during type 2 diabetes development by affecting insulin granule docked pool formation. J Pathol 2016, 238:109-119

19. Shen N, Gong T, Wang JD, Meng FL, Qiao L, Yang RL, Xue B, Pan FY, Zhou XJ, Chen HQ, Ning W, Li CJ: Cigarette smokeinduced pulmonary inflammatory responses are mediated by EGR1/GGPPS/MAPK signaling. Am J Pathol 2011, 178:110-118

20. Shen N, Shao Y, Lai SS, Qiao L, Yang RL, Xue B, Pan FY, Chen HQ, Li CJ: GGPPS, a new EGR-1 target gene, reactivates ERK 1/2 signaling through increasing Ras prenylation. Am J Pathol 2011, 179: $2740-2750$

21. Xu B, Chen C, Chen H, Zheng SG, Bringas P Jr, Xu M, Zhou X, Chen D, Umans L, Zwijsen A, Shi W: Smad1 and its target gene Wif1 coordinate BMP and Wnt signaling activities to regulate fetal lung development. Development 2011, 138:925-935

22. Fitzgerald ML, Xavier R, Haley KJ, Welti R, Goss JL, Brown CE, Zhuang DZ, Bell SA, Lu N, McKee M, Seed B, Freeman MW: ABCA3 inactivation in mice causes respiratory failure, loss of pulmonary surfactant, and depletion of lung phosphatidylglycerol. J Lipid Res 2007, 48:621-632

23. Shen N, Yu X, Pan FY, Gao X, Xue B, Li CJ: An early response transcription factor, Egr-1, enhances insulin resistance in type 2 diabetes with chronic hyperinsulinism. J Biol Chem 2011, 286:14508-14515
24. Glasser SW, Burhans MS, Eszterhas SK, Bruno MD, Korfhagen TR: Human SP-C gene sequences that confer lung epithelium-specific expression in transgenic mice. Am J Physiol Lung Cell Mol Physiol 2000, 278:L933-L945

25. Bahadue FL, Soll R: Early versus delayed selective surfactant treatment for neonatal respiratory distress syndrome. Cochrane Database Syst Rev 2012, 11:CD001456

26. Leung-Pineda V, Gronowski AM: Biomarker tests for fetal lung maturity. Biomark Med 2010, 4:849-857

27. Morimoto M, Kopan R: rtTA toxicity limits the usefulness of the SP-C-rtTA transgenic mouse. Dev Biol 2009, 325:171-178

28. Ridsdale R, Post M: Surfactant lipid synthesis and lamellar body formation in glycogen-laden type II cells. Am J Physiol Lung Cell Mol Physiol 2004, 287:L743-L751

29. Warburton D, Gauldie J, Bellusci S, Shi W: Lung development and susceptibility to chronic obstructive pulmonary disease. Proc Am Thorac Soc 2006, 3:668-672

30. Iber D, Menshykau D: The control of branching morphogenesis. Open Biol 2013, 3:130088

31. Bellusci S, Grindley J, Emoto H, Itoh N, Hogan BL: Fibroblast growth factor 10 (FGF10) and branching morphogenesis in the embryonic mouse lung. Development 1997, 124:4867-4878

32. Yamasaki M, Miyake A, Tagashira S, Itoh N: Structure and expression of the rat mRNA encoding a novel member of the fibroblast growth factor family. J Biol Chem 1996, 271:15918-15921

33. Ramasamy SK, Mailleux AA, Gupte VV, Mata F, Sala FG, Veltmaat JM, Del Moral PM, De Langhe S, Parsa S, Kelly LK, Kelly R, Shia W, Keshet E, Minoo P, Warburton D, Bellusci S: Fgf10 dosage is critical for the amplification of epithelial cell progenitors and for the formation of multiple mesenchymal lineages during lung development. Dev Biol 2007, 307:237-247

34. El Agha E, Bellusci S: Walking along the fibroblast growth factor 10 route: a key pathway to understand the control and regulation of epithelial and mesenchymal cell-lineage formation during lung development and repair after injury. Scientifica 2014, 2014:538379

35. Mailleux A, Tefft D, Ndiaye D, Itoh N, Thiery JP, Warburton D, Bellusci S: Evidence that SPROUTY2 functions as an inhibitor of mouse embryonic lung growth and morphogenesis. Mech Dev 2001, 102:81-94

36. Metzger RJ, Klein OD, Martin GR, Krasnow MA: The branching programme of mouse lung development. Nature 2008, 453:745-750

37. Sekine K, Ohuchi H, Fujiwara M, Yamasaki M, Yoshizawa T, Sato T, Yagishita N, Matsui D, Koga Y, Itoh N, Kato S: Fgf10 is essential for limb and lung formation. Nat Genet 1999, 21:138-141

38. Arman E, Haffner-Krausz R, Gorivodsky M, Lonai P: Fgfr2 is required for limb outgrowth and lung-branching morphogenesis. Proc Natl Acad Sci U S A 1999, 96:11895-11899

39. Zhu W, Nelson CM: PI3K regulates branch initiation and extension of cultured mammary epithelia via Akt and Rac1 respectively. Dev Biol 2013, 379:235-245

40. Zhu W, Nelson CM: PI3K signaling in the regulation of branching morphogenesis. Biosystems 2012, 109:403-411

41. Farnsworth CC, Gelb MH, Glomset JA: Identification of geranylgeranyl-modified proteins in HeLa cells. Science 1990, 247 : 320-322

42. Danopoulos S, Krainock M, Warburton D, Al Alam D: Rac1 plays an important role in lung morphogenesis through regulation of canonical Wnt signaling D23 pediatric rare diseases and lung development. Am Thorac Soc 2015:A5477-A 